\title{
The metabolic response to cancer
}

\author{
BY JOHAN GELIN AND KENT LUNDHOLM \\ Department of Surgery, Institution I and II, University of Göteborg, Sahlgrenska Hospital, \\ Göteborg, Sweden
}

The syndrome of cachexia is characterized by anorexia and profound losses of host tissues and constitutes one of the most common paraneoplastic conditions seen with cancer. Furthermore, cachexia is associated with a considerable mortality and morbidity but its origin is still obscure and cachexia remains a multi-factorial problem. A better understanding of the cause of the cachectic process and a reversal of its deleterious effects would improve not only the quality of life, but also life expectancy for the cancer patient. Weight loss occurs as a consequence of increased energy expenditure and a failure to compensate for this increased energy demand; weight loss is also aggravated by a cancer-associated anorexia. The mechanisms behind the increased energy expenditure remain to be fully elucidated but involve an altered intermediary metabolism including increased protein turnover, increased fat oxidation and gluconeogenesis.

\section{ENERGY EXPENDITURE}

It has been amply demonstrated that both resting and non-resting energy expenditure contribute to weight loss in patients with solid tumours (Warnold et al. 1978). In a recent study of 214 patients in our laboratory, it was possible to demonstrate that elevated energy expenditure preceded significant weight loss in unselected patients with solid tumours, suggesting that elevation in metabolism may be a cause of cachexia rather than a consequence (Hyltander et al. 1991). These studies have also pointed to the possibility that adrenergic mechanisms are involved in promoting this increased metabolism (Drott et al. 1987) and heart rate was a powerful factor in predicting alterations in resting energy expenditure. Such studies have revealed both an increased sensitivity and reactivity to adrenergic agonists in cancer patients (Drott et al. 1989) and a significantly higher urinary excretion of catecholamines and glucocorticoids in comparison with matched weightlosing patients without malignancy (Drott et al. 1988).

\section{CYTOKINES AND STRESS HORMONES}

A combination of mediators, both stress hormones and cytokines, are involved in the acute-phase response to various tissue injuries and neoplasia. What the causes and metabolic effects of the different cytokines are in a given pathological setting is difficult to discern, but there is a close link between the classical hormone system and the release of cytokines in the acute stress situation. This can be illustrated by the systemic release of glucocorticoids from the adrenal cortex following administration of cytokines (Fig. 1). The cytokines interleukin-1 (IL-1), tumour necrosis factor (TNF) and interleukin-6 (IL-6) have all been shown to stimulate the release of steroids either by a direct effect on the adrenal cortical cells to secrete glucocorticoids, or by an action on the hypothalamus 


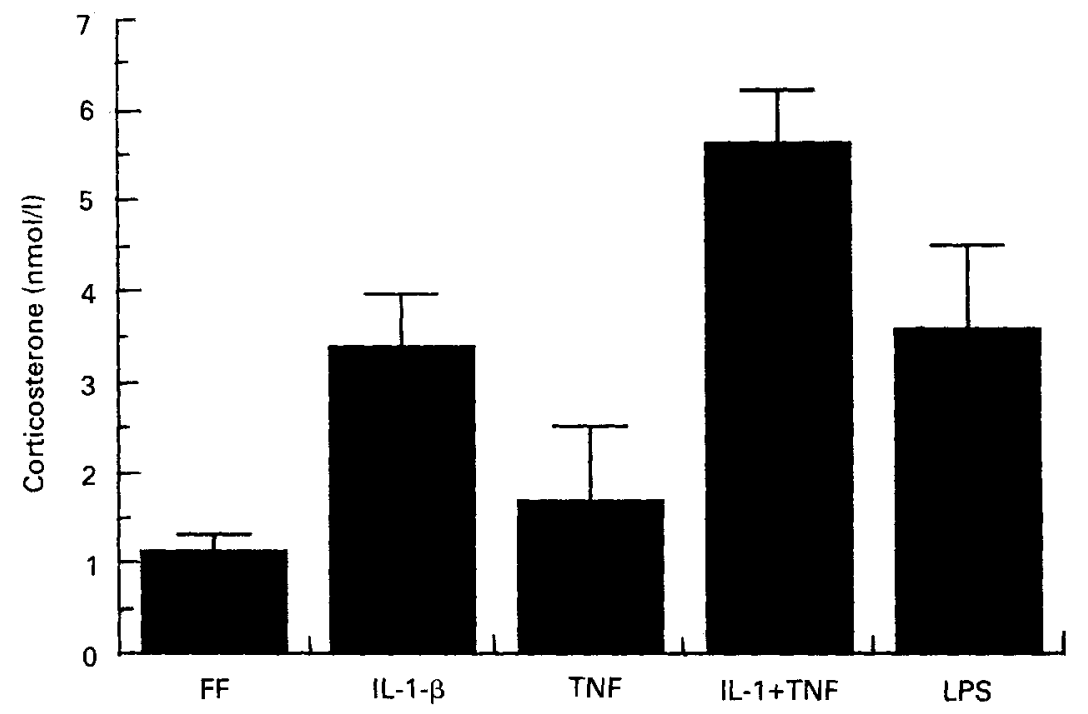

Fig. 1. Urinary corticosterone excretion in healthy mice administered interleukin-1 (IL-1; 420 ng/d), tumour necrosis factor (TNF; $450 \mathrm{ng} / \mathrm{d}$ ), combination IL-1 and TNF $(210+225 \mathrm{ng} / \mathrm{d})$ and lipopolysaccharide (LPS). Cytokines were administered as twice daily injections and LPS once daily. Urine was collected for $48 \mathrm{~h}$ in metabolism cages and corticosterone was estimated by a radioimmunoassay technique. Cytokine administration, and in particular IL-1, elaborated a significant adrenal cortical activity demonstrated as corticosteroid excretion. Values are means with their standard errors represented by vertical bars. FF, freely fed controls.

to release corticotrophin-releasing factor $(\mathrm{CRF})$, thus stimulating the pituitary to secrete adrenocorticotrophic hormone $(\mathrm{ACTH})$ to stimulate the adrenal cortex to secrete glucocorticoids. The subsequent increase in circulating steroids may regulate further glucocorticoid secretion by a negative feed-back system in vivo.

By administering physiological doses of recombinant IL-1 and TNF to healthy mice and collecting urine to determine corticosterone levels, increased steroid concentrations equivalent to the concentrations reached subsequent to lipopolysaccharide (LPS) administration (Gelin et al. 1992) could be demonstrated. This shows the close relationship between cytokine polypeptide factors and the endocrine system. The metabolic effects seen after tissue injury is, thus, probably in part explained by the effects that cytokines exert on the endocrine system.

\section{CYTOKINES AND CACHEXIA}

The current interest in cytokines as regards cachexia is the recognition of the similar host metabolic response evoked by various types of tissue injuries. Infections, inflammation and sepsis are all conditions that may ultimately lead to cachexia if prolonged and left untreated. The similarity in host response to these different types of insult suggests the existence of common mediators. Prolonged exogenous administration of TNF, IL-1 and IL-6 to experimental animals produces anorexia, loss of body-weight and an acute-phase reaction in the liver, which are all reactions typically seen in cancer disease. Furthermore, evidence is now beginning to emerge that TNF, IL-1 and perhaps IL-6 are 
produced endogenously by the tumour itself. Studies employing a sarcoma model have demonstrated expression of mRNA for both TNF and IL-1 and, furthermore, the presence of TNF protein in the tumour cells (Lönnroth et al. 1990). These results clearly demonstrate the ability of some tumour cells to elaborate these cytokines locally. Studies on human specimens by Balkwill and colleagues (Naylor et al. 1990) utilizing in situ hybridization techniques have confirmed TNF production in colonic and ovarian cancers. These findings agree well with the concept that TNF and IL-1 act primarily locally as paracrine or autocrine mediators. One prime function for this local endogenous TNF and IL-1 may be to induce the production of IL- 6 which may be a circulating messenger acting as an endocrine hormone (Jablons et al. 1989). IL-6 gives rise to an hepatic acute-phase response and furthermore, IL- 6 has been shown to induce anorexia (Busbridge et al. 1989). Hence, local production of TNF and IL-1 could clearly contribute to the development of cachexia, perhaps in some conditions through IL-6.

Much promise was initially hoped for TNF as an anti-tumour therapeutic agent (Balkwill et al. 1990). This proposition was based on the observation that TNF was cytotoxic or cytostatic to certain cell lines. TNF has subsequently been introduced in several clinical trials either alone or in combination with other agents in immunotherapy. The clinical results though have proved disappointing, with poor response rates and a high frequency of alarming side effects. Considering the recent demonstration of endogenous production of cytokines in the tumour-bearing situation and the physiological role of TNF and IL-1 as growth factors for normal cellular events, it has been proposed that both TNF and IL-1 may act as tumour growth factors. To test this hypothesis, attempts were made to block the biological actions of TNF and IL-1 by administering antibodies against the TNF molecule and the IL-1 receptor in tumourbearing animals. These experiments improved the animals' food intake along with tumour progression and, most surprising, caused a tumour growth inhibitory effect (Sherry et al. 1989; Gelin et al. 1991a). These in vivo effects were confirmed by growing these tumour cells in culture. These cells have previously been demonstrated to constitutively elaborate TNF and IL-1 (Lönnroth et al. 1990). Supplementing the culture medium with antibodies against IL-1 and TNF produced a cytostatic effect. When recombinant TNF or IL-1 was added to the culture medium, these cytokines acted as growth factors stimulating tumour growth. These findings may be contradictory to the prevailing opinion as to the effects of TNF and IL-1 when utilized as therapeutic agents.

Natural inhibitors that block the biological action of IL-1 and TNF have now been recognized and recently a receptor antagonist to IL-1 (IL-1ra) was cloned (Carter et al. 1990). It will be interesting to evaluate the effect of an administered endogenous antagonist to tumour-bearing animals.

\section{CYTOKINE INTERACTION}

The emerging picture of the role of cytokines in normal physiology and in disease states demonstrates a complex situation where agonist action is tightly controlled by natural antagonists. In addition, cytokines typically induce the synthesis of other cytokines that may have either synergistic or antagonistic effects. It follows from this complex biological setting that it is not possible to predict the biological outcome of a single cytokine since cytokine activity is dependent on the local environment of other growth factors. 


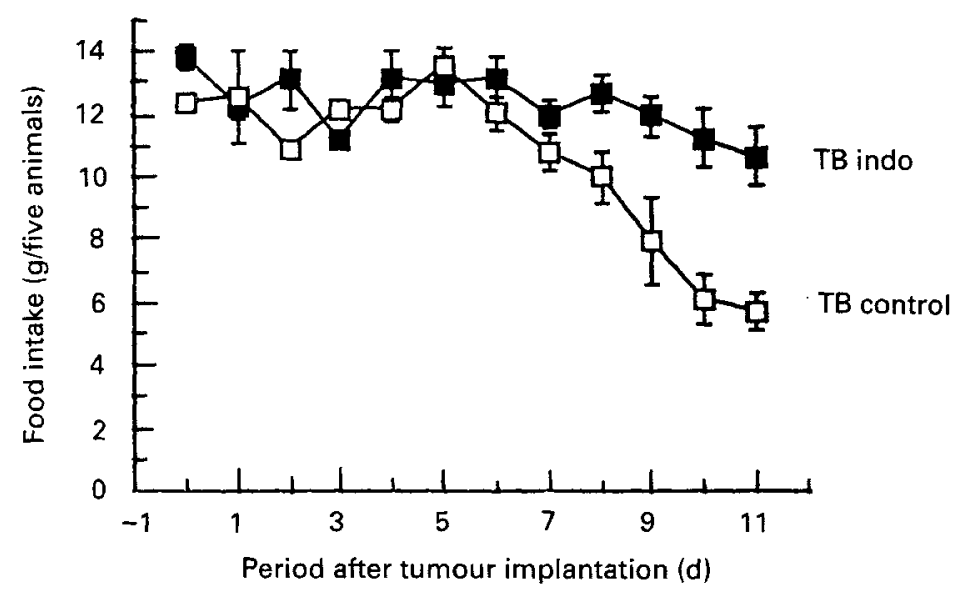

Fig. 2. Food intake in tumour-bearing (TB) animals treated with indomethacin (indo; $1 \mathrm{mg} / \mathrm{kg}$ per d) on a daily basis. This tumour model is well suited to study the effects of anorexia which develops on day 6 after tumour inoculation (TB control), when the tumour just becomes palpable. The anorexia is progressive with tumour growth. Indo maintains food consumption at near normal intake (TB indo). Indo also produces tumour inhibition, an effect that is clearly not substrate dependent (Fig. 1; Gelin et al. 1991b). Values are means with their standard errors represented by vertical bars.

\section{ANOREXIA AND TUMOUR GROWTH}

One of the main concerns in cancer management, and for that matter cancer treatment, is the associated anorexia. Although detrimental to the cancer patient, loss of appetite may be looked upon as an appropriate adaptation to the tumour-bearing state. The importance of feeding in maintenance of normal tumour cell cycle dynamics was recently demonstrated by the observation that starvation leads to an actual slowing of tumour growth and, thus, partially inhibits tumour growth in the $G_{0}-G_{1}$ phase (Westin, 1990).

In our mouse tumour model, attempting to influence tumour growth by administering IL-1 and TNF on a continuous daily basis along with tumour progression produced anorexia and a reduction of tumour growth (Gelin et al. 1991b). This inhibition of tumour growth by IL-1 could, in its entirety, be explained by anorexia alone and this effect did not provide any survival advantage. These animals exhibited a very poor body composition despite dying with smaller tumours. The anorectic effect of cytokines may well account for previously observed positive treatment effects on tumour regression in experimental animals.

A key effector of cytokine activity is the prostaglandins (PG), particularly of the PGE type. By blocking $\mathrm{PGE}_{2}$ activity with indomethacin (cyclo-oxygenase inhibitor) several positive treatment effects could be noted in the tumour-bearing situation. Anorexia was markedly attenuated along with tumour progression (Fig. 2) and survival was greatly improved. Animals survive $150 \%$ of controls and at spontaneous death, their body composition was better preserved. Despite the improvement in food consumption, there was significant tumour inhibition that appears to be substrate independent (Fig. 3). The action of indomethacin may be to obviate cytokine effects or may possibly be a specific 


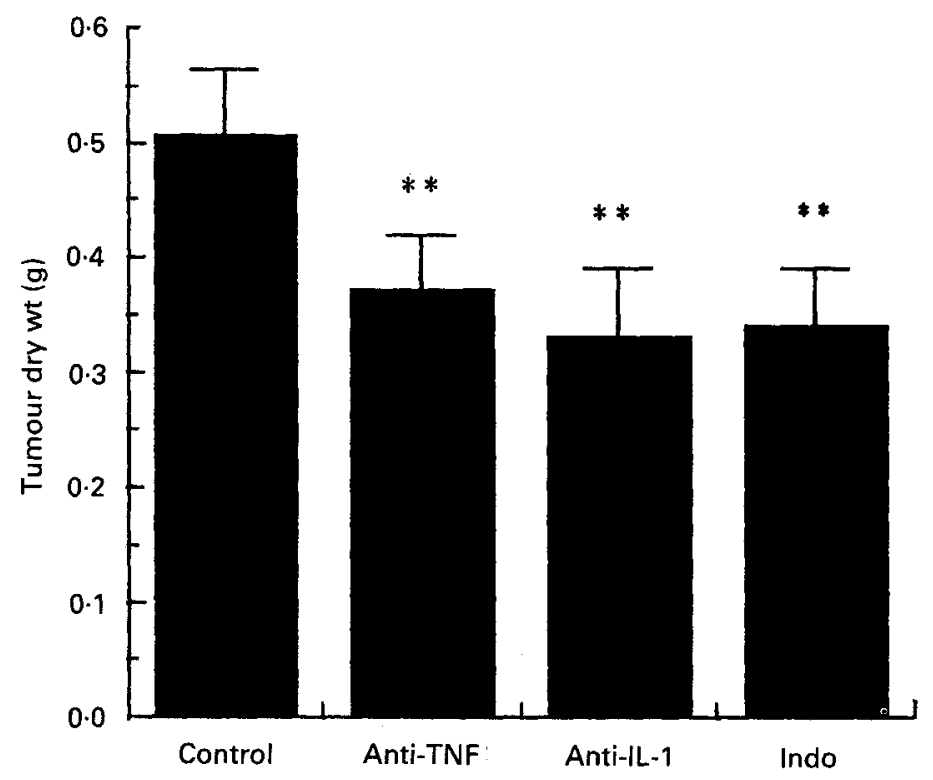

Fig. 3. Tumour dry weights in tumour-bearing animals. The animal groups were treated with either saline ( $9 \mathrm{~g}$ sodium chloride/l) or non-specific immunoglobulin $\mathrm{G}$ as controls, polyvalent immunoglobulin raised against the tumour necrosis factor (TNF) molecule (anti-TNF); monoclonal antibodies against the interleukin-1 (IL-1) receptor (anti-IL-1) or with indomethacin (indo). The tumours were dissected free on day 11 of tumour progression when the tumour comprises $100-150 \mathrm{~g} / \mathrm{kg}$ body-weight. Treatments with specific antibodies and indo produced significant tumour inhibition. Animals treated with indo showed a marked increase in survival and improved nutritional status at spontaneous death. Values are means with their standard errors represented by vertical bars. ${ }^{* *} P<0 \cdot 01$ compared to controls.

indomethacin effect. These positive treatment effects have been so encouraging as to initiate a clinical trial in patients with advanced cancers.

The term TNF may eventually prove to be a misnomer when its full biological activity as a growth factor is elucidated. Our own studies suggest that TNF and IL-1 act locally in or around the tumour and assume its known physiological role as a factor regulating cellular growth and angiogenesis and in these circumstances may act as a tumour growth factor. Blocking cytokine action by specific antibodies or administering indomethacin attenuated anorexia and improved the nutritional status of tumour-bearing animals in addition to inhibiting tumour growth.

Supported in parts by grants from the Swedish Cancer Society (93-B89-22XA, 2014-B8801XA, 2147-B89 04XA), the Medical Research Council (B89-17X-00536-25A, B89-17K08712-01A), Tore Nilson Foundation, Assar Gabrielsson Foundation (AB Volvo), Jubileumskliniken Foundation, Inga-Britt \& Arne Lundberg Research Foundation, Axel \& Margaret Ax:son Johnson Foundation, Swedish and Göteborg Medical Societies and the Medical Faculty, University of Göteborg.

\section{REFERENCES}

Balkwill, R. B., Naylor, S. N. \& Malek, S. (1990). Tumor necrosis factor as ari anticancer agent. European Journal of Cancer 26, 641-644. 
Busbridge, J., Dascombe, M. J., Hoopkins, S. \& Rothwell, N. J. (1989). Acute central effects of interleukin-6 on body temperature, thermogenesis and food intake in the rat. Proceedings of the Nutrition Society 38, $48 \mathrm{~A}$.

Carter, D. B., Deibel, M. R. Jr, Dunn, C. J., Tomich, C. S. C., Laborde, A. L., Slightom, J. L., Berger, A. E., Bienkowski, M. J., Sun, F. F., McEwan, R. N., Harris, P. K. W., Yem, A. W., Waszak, G. A., Chosay, J. G., Sieu, L. C., Hardee, M. M., Zurcher-Neely, H. A., Reardon, I. M., Heinrikson, R. L., Truesdell, S. E., Shelly, J. A., Eessalu, T. E., Taylor, B. M. \& Tracey, D. E. (1990). Purification, cloning, expression and biological characterization of an interleukin-1 receptor antagonist protein. Nature 344, 633-638.

Drott, C., Persson, H. \& Lundholm, K. (1989). Cardiovascular and metabolic response to adrenaline infusion in undernourished patients with and without cancer. Clinical Physiology 9, 427-439.

Drott, C., Svaninger, G. \& Lundholm, K. (1988). Increased urinary excretion of cortisol and catecholamines in malnourished cancer patients. Annals of Surgery 208, 645-650.

Drott, C., Waldenström, A. \& Lundholm, K. (1987). Cardiac sensitivity and responsiveness to $\beta$-adrenergic stimulation in experimental cancer and undernutrition. Journal of Molecular and Cellular Cardiology 19, $675-683$

Gelin, J., Andersson, C. \& Lundholm, K. (1991a). Effects of indomethacin, cytokines and cyclosporin A on tumor growth and the subsequent development of cancer cachexia. Cancer Research 51, 880-885.

Gelin, J., Moldawer, I., Iresjö, B. M. \& Lundholm, K. (1992). The role of the adrenals in the acute-phase response to interleukin 1 and tumor necrosis factor alpha. Journal of Surgical Research. (In the Press).

Gelin, J., Moldawer, L. L., Lönnroth, C., Sherry, B., Chizzonite, R. \& Lundholm, K. G. (1991b). The role of endogenous tumour necrosis factor $\alpha$ and Interleukin-1 for experimental tumour growth and the development of cancer cachexia. Cancer Research 51, 440-445.

Hyltander, A., Drott, C., Körner, U., Sandström, R. \& Lundholm, K. (1991). Elevated energy expenditure in cancer patients with solid tumours. European Journal of Cancer 27, 9-15.

Jablons, D. M., Mulé, J. J., McIntosh, J. K., Sehgel, P. B., May, L. T., Huang, C. M., Roenberg, S. A. \& Lotze, M. T. (1989). Interleukin-6/Interferon- $\beta_{2}$ as a circulating hormone. Journal of Immunology 142, $1542-1547$.

Lönnroth, C., Moldawer, L.. Gelin, J., Kindblom, L. G., Sherry, B. \& Lundholm, K. (1990). Tumor necrosis factor alpha and interleukin 1 alpha production in cachectic, tumor-bearing mice. International Journal of Cancer 46, 889-896.

Naylor, M. S., Stamp, G. W. H. \& Balkwill, F. R. (1990). Investigation of cytokine gene expression in human colorectal cancer. Cancer Research 50, 4436-4440.

Sherry, B. A., Gelin, J., Fong, Y., Marano, M., Wei, H., Cerami, A., Lowry, S. F. \& Lundholm, K. G. (1989). Anticachectin/tumor necrosis factor- $\alpha$ antibodies attenuate development of cachexia in tumor models. FASEB Journal 3, 1956-1962.

Warnold, I., Lundholm, K. \& Scherstén, T. (1978). Energy balance and body composition in cancer patients. Cancer Research 38, 1801-1807.

Westin, T. (1990). Ornithine decarboxylase activity in malignant tumors. An experimental and clinical study with reference to cell proliferation and nutrition. Thesis, University of Göteborg. 